\title{
A New Measure of Binge Drinking: Prevalence and Correlates in a Probability Sample of Undergraduates
}

\author{
James A. Cranford, Sean Esteban McCabe, and Carol J. Boyd
}

\begin{abstract}
Background: A standard measure defines binge drinking as the consumption of 5 or more drinks in a row for men ( 4 or more drinks for women) on at least 1 occasion during the past 2 weeks. A revised operational definition of binge drinking was developed by the National Institute on Alcohol Abuse and Alcoholism in 2004 and incorporated the duration of the drinking episode in addition to the quantity of alcohol consumed. This study compares the standard and new binge measures for overall and subgroup prevalence rates; associations with gender, race/ethnicity, and age of drinking onset; and associations with negative drinking consequences.

Methods: A probability sample of 4,580 randomly selected college students $(50.3 \%$ female, $M$ age $=19.9, S D=2.0$ ) at a large Midwestern university in the United States completed a Web-based survey of alcohol and other drug use. Participants reported on past 2-week binge drinking using the standard measure and past-year binge drinking using the new measure.

Results: The longer past-year time frame of the new measure yielded a higher prevalence estimate of binge drinking $(63.6 \%)$ compared with the 2-week standard measure $(53.2 \%)$. Approximately $9.9 \%$ of those who were classified as binge drinkers using the 2-week standard measure were classified as non-binge drinkers using the new measure specification of a 2-hour duration for the drinking episode. The past-year new binge measure was positively associated with negative drinking consequences even when the 2-week measure was statistically controlled.

Conclusions: Using a longer time frame and incorporating the duration of the drinking episode, the new measure of binge drinking appears to capture an important element of risky alcohol involvement in college students that is not fully assessed by the standard measure.
\end{abstract}

Key Words: Binge Drinking, Gender and Race Differences in Drinking, Age of Drinking Onset, Negative Drinking Consequences, College Students.

$\mathrm{H}$ EAVY EPISODIC OR "binge" drinking among college students has been identified as an important public health concern (Ham and Hope, 2003; Hingson et al., 2005; Wechsler et al., 2002). Despite many public health efforts to reduce this risky behavior, levels of collegiate binge drinking have remained remarkably stable over the past decade, with approximately $40 \%$ of college students reporting this behavior (Johnston et al., 2005; Wechsler et al., 2002). Research has shown that when using the standard binge measure of consuming 5 or more drinks in a row for men (4 or more drinks for women) per occasion within 2 weeks (Wechsler et al., 1994), the prevalence of binge drinking is higher among college

From the Substance Abuse Research Center, University of Michigan, Ann Arbor, Michigan.

Received for publication September 13, 2005; accepted July 14, 2006.

This work was supported by a research grant DA 018239 (PI: SEM) from the National Institute on Drug Abuse and a research grant AA 015275 (PI: SEM) from the National Institute on Alcohol Abuse and Alcoholism, National Institutes of Health.

Reprint requests: James A. Cranford, PhD, Substance Abuse Research Center, University of Michigan, 2025 Traverwood Drive, Suite C, Ann Arbor, MI 48105-2194; Fax: 734-998-6508; E-mail: jcranfor@umich.edu

Copyright (C) 2006 by the Research Society on Alcoholism.

DOI: 10.1111/j.1530-0277.2006.00234.x students compared with their non-college-attending peers (Dawson et al., 2004; Office of Applied Studies, 2003; Slutske, 2005).

The high prevalence of binge drinking is particularly alarming in light of the numerous alcohol-related problems associated with this behavior. College students who binge drink are at greater risk for poor academic performance, risky sexual behavior, driving after drinking, and physical injury (Perkins, 2002; Wechsler and Isaac, 1992; Wechsler et al., 1994), and a recent review indicated that the number of alcohol-related deaths among college students 18 to 24 years of age increased from approximately 1,600 in 1998 to more than 1,700 in 2001 (Hingson et al., 2005). Heavy alcohol use among college students is also associated with negative consequences for other people and to the surrounding community (Perkins, 2002; Task Force of the National Advisory Council on Alcohol Abuse and Alcoholism, 2002).

The criterion of 5 or more drinks on a drinking occasion as an indicator of problem drinking was first proposed by Cahalan et al. (1969; also see Cisin and Cahalan, 1968) as a threshold for evaluating the negative consequences associated with drinking. Starting in 1975, investigators from the Monitoring the Future Study began asking participants how often they had consumed 5 drinks in a row during the past 2 weeks and later referred to this 
behavior as "binge drinking" (O'Malley et al., 1984). In the early 1990s, the College Alcohol Study (CAS; Wechsler et al., 1994) also used the term "binge drinking" and introduced the gender-specific measure, which defined binge drinking for women as 4 drinks in a row during the past 2 weeks.

The operational definition of binge drinking based on consumption of $5 / 4$ drinks on at least 1 occasion in the past 2 weeks has proven controversial (DeJong, 2001; Lange and Voas, 2001; Perkins et al., 2001; Wechsler and Austin, 1998; Wechsler and Nelson, 2001). Wechsler and Austin (1998) noted that clinicians expressed concern because the term "binge" had been previously used to refer to the prolonged drinking bouts of alcoholic patients. Dimeff et al. (1995) argued that heavy episodic drinking is normative on college campuses and that the term "binge drinking" implied that this behavior was pathological. Dawson et al. (2004) pointed out that the use of a 2-week reference period may underestimate the prevalence of infrequent binge drinking. Also, research by Weingardt et al. (1998) suggested that the standard binge criteria have high sensitivity but low specificity. In addition, the standard definition of binge drinking has been criticized for failure to specify the duration of the drinking episode. For example, Lange and Voas (2001) argued that, because it ignores duration of consumption, the standard definition does not map onto levels of blood alcohol concentration (BAC) that are associated with intoxication (also see Beirness et al., 2004, 2005; Naimi and Brewer, 2005; Nelson and Xuan, 2005).

In an attempt to address these concerns, the National Institute on Alcohol Abuse and Alcoholism (NIAAA) convened a task force to develop a definition of binge drinking for future research efforts. In February 2004, the NIAAA National Advisory Council approved the following definition: "A 'binge' is a pattern of drinking alcohol that brings $\mathrm{BAC}$ to about 0.08 gram-percent or above. For the typical adult, this pattern corresponds to consuming 5 or more drinks (male), or 4 or more drinks (female), in about 2 hours" (NIAAA, 2004). This new definition incorporates the duration of the drinking episode in addition to the quantity of alcohol consumed to define binge drinking.

In this article, we report results from a large-scale cross-sectional study of college students that included a measure based on this new operational definition of binge drinking, using a past 12-month time frame, along with a measure based on what Weingardt et al. (1998) refer to as the "standard" binge criteria of 5/4 drinks in a row during the past 2 weeks. We hypothesized that the past-year new and the 2-week standard measures of binge drinking would be highly correlated, that prevalence rates of binge drinking would be higher using the past-year new measure (based on its longer time frame), and that the specification of a 2-hour duration in the new measure would decrease the absolute number of participants classified as binge drinkers. Because previous work using the 2-week standard measure has shown elevated rates of binge drinking among males compared with females (Wechsler et al., 2000), Whites and Hispanics compared with Blacks and Asians (Paschall et al., 2005; Wechsler et al., 2000), and those with an early age of drinking onset (ADO; Muthen and Muthen, 2000), we also compared prevalence rates of binge drinking by gender, race, and ADO using the 2 measures. Here, it was hypothesized that the pattern of prevalence rates would be similar for the 2 measures, but that across groups the pastyear new measure would show higher overall rates of binge drinking. Finally, it was hypothesized that the past-year new and 2-week standard measures assess different dimensions of risky drinking. Specifically, because of its longer time frame and its use of a 2-hour window for the drinking episode, the new past-year measure may capture a dimension of risky drinking that is not fully assessed by the standard measure. Accordingly, it was predicted that the new measure would independently predict alcoholrelated problems and potential alcohol abuse, even when the standard measure was statistically controlled.

\section{MATERIALS AND METHODS}

This research was based on a larger study of college students conducted during January and February 2005, drawing on a total undergraduate population of 20,138 full-time students $(10,339$ women and 9,799 men) at a large Midwestern university. A probability sample of 5,389 full-time undergraduate students was randomly selected from the total undergraduate population. Additionally, we oversampled 652 Hispanic, 634 Black, and 244 Asian undergraduate students to produce reliable prevalence estimates for these racial/ethnic groups

After receiving Human Subjects Review Board approval to conduct the study, the entire sample was mailed a prenotification letter with 2 dollars enclosed that described the study and invited students to self-administer a Web survey by using a URL address and unique password. The 2 dollar prepayment, which was described as a token of appreciation for participation in the study, was selected based on pilot work showing that its inclusion resulted in a higher response rate and a more representative sample and increased the perceived legitimacy of the subsequent e-mail invitation. The study website was active for approximately 1 month. Following the mailed prenotification letter, nonrespondents were sent an invitation e-mail and up to 4 reminder e-mails with the URL address and unique password to facilitate participation in the Web survey. The 4 reminder emails were sent at 3,8,17, and 29 days after the initial e-mail invitation. The Web survey was maintained on an Internet site running under the secure socket layer protocol to ensure privacy and security. Active consent was provided online. By participating in the survey, students became eligible for a sweepstakes that included cash prizes, travel vouchers, field passes to athletic events, and iPods. The final response rate was $66.2 \%$, which exceeded the average response rate for recent national college-based alcohol and other drug studies that used mail surveys (Wechsler et al., 2002).

The sample included 4,580 undergraduate students $(3,639$ from the random sample and 941 from the oversample). The unweighted sample consisted of $54.7 \%$ women, $45.3 \%$ men, $53.6 \%$ White, $13.3 \%$ Asian, $12.8 \%$ Black, $12.3 \%$ Hispanic, 0.6\% Native American, and $7.4 \%$ from other racial categories. The mean (SD) age of students in the sample was 19.9 (2.0) years old, and the breakdown by class year was $27.8 \%$ freshmen, $24.2 \%$ sophomores, $23.1 \%$ juniors, and $25.0 \%$ seniors. A sample weight variable was created to account for oversampling of racial/ethnic minorities and a higher 
percentage of women who responded to the Web survey compared with the percentage of women in the undergraduate student population. Data were weighted for analyses of the overall sample to increase the representativeness of our results. The weight variable was centered (normalized) to ensure that the sample size remained the same after weighting. As a result, our sample closely resembled the overall student population on demographic characteristics.

\section{Measures}

The Web survey included questions on a wide range of topics, including alcohol, drug and tobacco use, gambling, and motivations. Below we describe the measures used in the present study.

Past 2-Week Binge Drinking. Participants were asked "Over the past 2 weeks, how many occasions have you had [5 (male)/4 (female)] or more drinks in a row?" Responses were made using a 6-point scale consisting of the following response options: none (1), once (2), twice (3), 3 to 5 times (4), 6 to 9 times (5), and 10 or more times (6). A drink was defined as a glass of wine, a bottle of beer or wine cooler, or a shot of liquor straight or in a mixed drink. A genderspecific measure of past 2-week binge drinking was constructed by recoding scores on the frequency of binge drinking item, so that participants who reported at least 1 binge drinking episode in the past 2 weeks were classified as "past 2 -week binge drinkers." This coding scheme is based on the work of Wechsler et al. (1995). We refer to this as the "2-week standard measure of binge drinking."

Past-Year Maximum Drinks in a 2-Hour Period. This variable was assessed by asking students "What is the greatest number of drinks you consumed in a 2-hour period during the past 12 months?" Responses ranged from 0 to 35 . Cases with values that were 3 or more standard deviations above the mean (i.e., a score of 19 or higher, $1 \%$ of all cases) were defined as outliers based on the criteria outlined by Stevens (1996). Values for these outlier cases were set to the maximum value of 19. All analyses were conducted with and without this recode for the outlying cases. No differences between the 2 sets of results were observed.

Past-Year Binge Drinking: NIAAA Measure. The item concerning maximum drinks in a 2-hour period in the previous 12 months was used to categorize past-year binge drinkers. Based on the definition advanced by the NIAAA National Advisory Council (NIAAA, 2004), participants were classified as "past-year binge drinkers" if they reported consuming 5 or more drinks (male) or 4 or more drinks (female) at least 1 time in a 2-hour period during the past 12 months. We refer to this as the "past-year new measure of binge drinking." Although the NIAAA definition does not specify a reference period, we asked about alcohol consumption during the past year based on the NIAAA Task Force on Recommended Sets of Alcohol Consumption Questions (NIAAA, 2002), which indicated that a past-year reference period allows for the assessment of infrequent drinkers. Other alcohol researchers have suggested that a past-year reference period may be optimal for linking alcohol consumption and alcohol-related problems (Dawson, 2003; Dawson and Room, 2000; Sobell and Sobell, 2003). However, we note that the use of a past-year reference period is in contrast to previous studies, which have typically examined college student binge drinking during the past 2 weeks (e.g., Johnston et al., 2004; Wechsler et al., 2002).

Age of Drinking Onset. Participants were asked "What grade were you in when you first started drinking alcohol (more than just a few sips)?" Responses were made using a 6-point scale consisting of the following response options: Grades $\mathrm{K}$ to $4(n=15,0.4 \%)$, Grades 5 to $6(n=41,1 \%)$, Grades 7 to $8(n=226,5.7 \%)$, Grades 9 to 10 $(n=880,22.0 \%)$, Grades 11 to $12(n=1,448,36.3 \%)$, and college $(n=1,381,34.6 \%)$. To increase sample sizes, Grades K to 4 , Grades 5 to 6 , and Grades 7 to 8 were collapsed into 1 category. This recode increased the sample size for the group of participants who began drinking at around age 14 or younger (i.e., Grade K to 8), a cutoff that has been identified as predictive of later alcohol problems (Hingson et al., 2000; York et al., 2004).

Past-Year Negative Alcohol Consequences. We included 11 items adapted from the College Alcohol Study (Harford et al., 2002; Wechsler et al., 1994) and the Core Alcohol and Drug Survey (Presley et al., 1996) to assess negative drinking consequences. Participants were asked to indicate how often they had experienced several negative alcohol-related consequences during the past year (e.g., performed poorly on a test or important project; missed a class or work due to drinking; had unplanned sex). Responses were made using a 5-point scale consisting of the following response options: no occasions (1), 1 to 2 occasions (2), 3 to 5 occasions (3), 6 to 9 occasions (4), and 10+ occasions (5). Because all of these items had a marked positive skew, they were recoded into dichotomous variables $(0=$ consequence not experienced, $1=$ consequence experienced) (see Table 4 for complete listing of items).

The CAGE Questionnaire. The CAGE questionnaire is a widely used 4-item screening measure for alcohol problems (Ewing, 1984; Maisto and Saitz, 2003). Although the CAGE was originally designed as a screen for lifetime alcohol problems (Mayfield et al., 1974), we adapted the CAGE items to assess alcohol problems in the past year. Participants were asked to indicate how often, during the past 12 months, they had experienced the following: (1) felt that you should cut down your drinking, (2) been annoyed by people criticizing your drinking, (3) felt guilt or remorse after drinking, and (4) had a drink first thing in the morning as an "eye opener" or to get rid of a hangover. Responses were made using a 5-point scale consisting of the following response options: no occasions (1), 1 to 2 occasions (2), 3 to 5 occasions (3), 6 to 9 occasions (4), and $10+$ occasions (5). Scores for each item were then recoded so that $0=$ no occasions in the past 12 months and $1=$ at least 1 occasion in the past 12 months, and a total score ranging from 0 to 4 was computed by summing across items. Scores of 2 or higher are considered clinically significant and indicate the need for further assessment (Boyd et al., 2003; Connors and Volk, 2003).

\section{Statistical Analysis Plan}

Initial analyses examined the overall prevalence rates for the 2-week standard and past-year new measures of binge drinking, along with their bivariate association. Prevalence rates by subgroups defined by gender, race, and ADO were estimated using both measures. Multiple logistic regression analyses were used to examine the associations between gender, race, and ADO and both binge drinking measures. Finally, multiple logistic regression analysis was used to test the hypothesis that the past-year new binge measure would be associated with negative drinking consequences and potential alcohol abuse even when past 2 -week binge drinking, gender, race/ethnicity, and ADO were statistically controlled. In these analyses, all variables were entered simultaneously into the multiple logistic regression equation predicting each dichotomously scored negative drinking consequence and the dichotomous variable reflecting potential alcohol abuse. The results from these analyses show the effect of past-year binge drinking on the odds of each negative drinking consequence when all other variables in the equation (i.e., past 2 weeks binge drinking, gender, race/ethnicity, and ADO) are held constant (see Agresti, 1996; Jaccard, 2001). All analyses were conducted with SAS version 9.1.3 using the PROC SURVEYFREQ and PROC SURVEYLOGISTIC steps for the analysis of data from complex survey sample designs, including those with unequal weighting (SAS Institute, 2004).

\section{RESULTS}

Except where indicated, all analyses were based on weighted data as described earlier. We also indicate analyses that 
were based on the full sample versus those based on pastyear drinkers $(n=3,856,86.2 \%$ of the sample).

\section{Overall Prevalence Rates and Bivariate Association Between Past 2-Week and 12-Month Binge Drinking}

For the full sample, the overall prevalence of past 2 -week binge drinking was $53.1 \%$. In contrast, the overall prevalence of past-year binge drinking was $63.6 \%$. These 2 prevalence rates were significantly different, McNemar's $\chi^{2}(1)=278.0, p<0.01$. As hypothesized, the 2 measures of binge drinking were strongly correlated, $\phi=0.63, p<0.01$. As can be seen in Table 1, there was a high degree of concordance between the 2 binge measures, and among those who indicated binge drinking at least once in the past year, $77.9 \%$ also reported at least 1 binge drinking episode in the past 2 weeks. The results in Table 1 also show that $22.1 \%$ of past-year binge drinkers did not report binge drinking in the past 2 weeks, suggesting that the longer past-year time frame of the new binge measure led to the categorization of more binge drinkers. However, as can be seen in Table 1 , the results also show that 9.9\% $(n=159)$ of those who indicated that they did not consume $4 / 5$ drinks in a 2-hour period during the past year responded that they did consume $4 / 5$ drinks "in a row" in the past 2 weeks. In other words, these participants were categorized as past 2-week binge drinkers, but they apparently did not consume $4 / 5$ drinks within a 2 -hour period. This finding suggests that the 2-hour window specified by the past-year new binge measure excluded those participants who consumed $4 / 5$ drinks over a span of time exceeding 2 hours, but who were categorized as "binge" drinkers by the 2-week standard measure.

\section{Prevalence Rates of Past 2-Week and 12-Month Binge Drinking by Gender, Race, and ADO}

To further compare the 2 binge measures, prevalence rates of past 2-week and past-year binge drinking were calculated for subgroups defined by gender, race, and ADO.

Table 1. Association Between Past-Year and Past 2-Week Binge Drinking

\begin{tabular}{lccc}
\hline & \multicolumn{3}{c}{ Past 2-week binge drinking } \\
\cline { 2 - 4 } Past year \\
binge drinking
\end{tabular}

Note: Rao-Scott $\chi^{2}(1)=1,751.8, \phi=0.63, p<0.01$. Analyses based on data weighted by gender and race/ethnicity. Cell percentages are based on row totals for past-year binge drinking.

aBased on the "new" NIAAA-recommended definition of binge drinking as the consumption of 5 or more drinks (male) or 4 or more drinks (female) in a 2-hour period at least 1 time during the past year.

"Based on the "standard" definition of binge drinking as the consumption of 5 or more drinks (male) or 4 or more drinks (female) in a row at least 1 time during the past 2 weeks.
Table 2. Prevalence of Past 2-Week and Past-Year Binge Drinking by Gender, Ethnicity, and ADO

\begin{tabular}{|c|c|c|}
\hline & Past 2-week binge drinking & Past-year binge drinking \\
\hline Overall $^{\mathrm{a}}$ & 53.1 & 63.6 \\
\hline \multicolumn{3}{|l|}{ Gender $^{a}$} \\
\hline Female & 52.3 & 63.0 \\
\hline Male & 54.1 & 64.3 \\
\hline \multicolumn{3}{|l|}{ Ethnicity $^{\mathrm{a}}$} \\
\hline \multicolumn{3}{|l|}{ Asian } \\
\hline Total & 33.2 & 45.5 \\
\hline Female & 29.4 & 41.8 \\
\hline Male & 36.7 & 49.0 \\
\hline \multicolumn{3}{|l|}{ Black } \\
\hline Total & 26.1 & 40.8 \\
\hline Female & 20.0 & 33.9 \\
\hline Male & 35.4 & 51.2 \\
\hline \multicolumn{3}{|l|}{ Hispanic } \\
\hline Total & 61.8 & 68.9 \\
\hline Female & 59.4 & 68.6 \\
\hline Male & 64.0 & 71.0 \\
\hline \multicolumn{3}{|l|}{ White } \\
\hline Total & 60.7 & 70.4 \\
\hline Female & 60.4 & 70.7 \\
\hline Male & 60.9 & 70.1 \\
\hline \multicolumn{3}{|l|}{ Other } \\
\hline Total & 45.2 & 51.6 \\
\hline Female & 49.2 & 53.5 \\
\hline Male & 42.4 & 50.3 \\
\hline \multicolumn{3}{|c|}{ Age of drinking onset ${ }^{\mathrm{b}}$} \\
\hline \multicolumn{3}{|l|}{ College } \\
\hline Total & 40.0 & 54.7 \\
\hline Female & 38.3 & 50.4 \\
\hline Male & 41.9 & 59.2 \\
\hline \multicolumn{3}{|c|}{ Grades 11 to 12} \\
\hline Total & 65.0 & 75.8 \\
\hline Female & 63.5 & 75.5 \\
\hline Male & 66.8 & 76.1 \\
\hline \multicolumn{3}{|c|}{ Grades 9 to 10} \\
\hline Total & 77.3 & 86.8 \\
\hline Female & 74.5 & 86.2 \\
\hline Male & 80.4 & 87.5 \\
\hline \multicolumn{3}{|c|}{ Grades $\mathrm{K}$ to 8} \\
\hline Total & 73.2 & 83.0 \\
\hline Female & 74.9 & 84.6 \\
\hline Male & 72.2 & 82.1 \\
\hline
\end{tabular}

Note. Analyses based on data weighted by gender and race/ethnicity. aPercentages based on overall $N=4,580$.

${ }^{b}$ Percentages based on $N=3,997$ lifetime alcohol users who were asked about age of drinking onset.

The results in Table 2 show that, similar to the pattern of overall prevalence rates for the 2 measures, the subgroup prevalence rates based on the past-year new measure were elevated compared with those based on the past 2-week standard measure. For both measures, prevalence rates were slightly higher for males compared with females, for Whites and Hispanics compared with Blacks and Asians, and for early compared with late onset drinkers.

\section{Gender, Race/Ethnicity, and Early ADO as Predictors of Past 2-Week and 12-Month Binge Drinking}

Having compared overall and subgroup prevalence rates for the 2 binge measures, we tested the hypothesis that gender, race/ethnicity, and early age of onset would be 
Table 3. Multiple Logistic Regression Analysis of Gender, Ethnicity, and Age of Drinking Onset as Predictors of Past 2-Week and Past-Year Binge Drinking

\begin{tabular}{|c|c|c|c|c|}
\hline \multirow[b]{2}{*}{ Predictor } & \multicolumn{2}{|c|}{ Past 2-week binge drinking } & \multicolumn{2}{|c|}{ Past-year binge drinking } \\
\hline & AOR & $95 \% \mathrm{Cl}$ & AOR & $95 \% \mathrm{Cl}$ \\
\hline \multicolumn{5}{|l|}{ Gender } \\
\hline Female & $-^{a}$ & & $-^{a}$ & \\
\hline Male & $1.18^{*}$ & 1.02 to 1.37 & $1.20^{*}$ & 1.03 to 1.41 \\
\hline \multicolumn{5}{|l|}{ Ethnicity } \\
\hline Black & $-^{a}$ & & $-^{a}$ & \\
\hline White & $3.55^{* *}$ & 2.80 to 4.50 & $2.72^{* *}$ & 2.18 to 3.41 \\
\hline Hispanic & $3.37^{* *}$ & 2.52 to 4.53 & $2.33^{* *}$ & 1.74 to 3.12 \\
\hline Asian & $1.48^{* *}$ & 1.10 to 1.20 & $1.34 *$ & 1.01 to 1.78 \\
\hline Other & $2.00^{* *}$ & 1.44 to 2.78 & $1.53^{* *}$ & 1.11 to 2.11 \\
\hline \multicolumn{5}{|c|}{ Age of drinking onset } \\
\hline College & - $^{a}$ & & $-^{a}$ & \\
\hline Grades 11 to 12 & $2.74^{* *}$ & 2.34 to 3.20 & $2.52^{* *}$ & 2.13 to 3.00 \\
\hline Grades 9 to 10 & $4.72^{* *}$ & 3.88 to 5.73 & $5.05^{* *}$ & 3.98 to 6.39 \\
\hline Grades $\mathrm{K}$ to 8 & $4.15^{* *}$ & 3.10 to 5.56 & $4.05^{* *}$ & 2.84 to 5.79 \\
\hline
\end{tabular}

Note. AOR, adjusted odds ratios from multiple logistic regression analyses with gender, race/ethnicity, and age of drinking onset as predictors of past 2-week and past-year binge drinking; 95\% Cl, 95\% confidence interval for the adjusted odds ratio. Analyses based on $n=3,997$ lifetime alcohol users who were asked about age of drinking onset. Data weighted by gender and race/ethnicity.

${ }^{\mathrm{a}}$ Reference group.

${ }^{*} p<0.05{ }^{* *} p<0.01$

differentially associated with past 2-week and past-year binge drinking. A series of multiple logistic regression analyses were conducted to assess the effects of gender, race/ethnicity, and $\mathrm{ADO}$ on the odds of past 2-week and past-year binge drinking. Four dummy variables were constructed to represent the information for the 5 -category race/ethnicity variable, and 3 dummy variables were created to represent the 4-category ADO variable. For gender, the reference group was females, for race/ ethnicity the reference group was Black, and for ADO the reference group was college. These groups were selected as reference groups because they had the lowest prevalence rates of past 2-week and past-year binge drinking.

The results presented in Table 3 showed that, controlling for race/ethnicity and ADO, the adjusted odds of past 2-week and past-year binge drinking were higher among males compared with females. For ethnicity, the results showed that, controlling for gender and ADO, the odds of past 2-week and past-year binge drinking were statistically significantly higher among Whites, Hispanics, and others compared with Blacks. The odds of past 2-week binge drinking were higher among Asians compared with Blacks, but this difference was not observed for the past-year binge measure. As can be seen in Table 3, the magnitude of the odds ratios (OR) for the associations between race/ethnicity and both measures of binge drinking was generally similar. Additional analyses showed (a) significantly higher odds for Whites compared with Asians on past 2-week $(\mathrm{OR}=2.39, p<0.01)$ and past-year binge drinking $(\mathrm{OR}=2.04, p<0.01)$ and (b) significantly higher odds for Hispanics compared with Asians on past 2-week $(\mathrm{OR}=2.28, p<0.01)$ and past-year binge drinking $(\mathrm{OR}=1.74, p<0.01)$. No other significant race/ethnicity differences were observed for either measure.

Early ADO also showed statistically significant associations with both measures of binge drinking. As can be seen in Table 3, controlling for gender and race/ethnicity, the odds of past 2-week and past-year binge drinking were significantly higher among those who reported drinking onset in middle school (Grades $\mathrm{K}-8$ ), early in high school (Grades 9-10), and later in high school (Grades 11-12) compared with those who began drinking in college. The ORs for the associations between ADO and both measures of binge drinking were very similar in magnitude. Additional analyses showed significantly higher odds for past 2-week $(\mathrm{OR}=1.52, p<0.01)$ and past-year $(\mathrm{OR}=1.61$, $p<0.01)$ binge drinking among those who reported drinking onset in Grades K to 8 compared with Grades 11 to 12 . We also observed significantly higher odds for past 2-week $(\mathrm{OR}=1.72, p<0.01)$ and past-year $(\mathrm{OR}=2.00, p<0.01)$ binge drinking among those who reported drinking onset in Grades 9 to 10 compared with Grades 11 to 12 . No differences in the odds of past 2-week or past-year binge drinking were observed between those with an ADO of Grades 9 to 10 compared with those with an ADO of Grades $\mathrm{K}$ to 8 . Taken together, these findings indicate that gender, race/ethnicity, and ADO had similar associations with the 2-week standard and past-year new binge measures.

\section{Associations Between Past-Year Binge Drinking and Negative Drinking Consequences}

As a further comparison between the 2-week standard and past-year new binge measures, the associations between past-year binge drinking and each of the negative drinking consequences, including potential alcohol abuse, were examined in a series of multiple logistic regression analyses. In the first set of analyses, past-year binge drinking was tested as a predictor of each negative drinking consequence after controlling for gender, race/ethnicity, and ADO. As can be seen in the top panel of Table 4, past-year binge drinking was statistically significantly associated with all 12 of the negative drinking consequences. The results showed particularly strong associations between past-year binge drinking and (1) blackouts (amnesia), (2) being hurt or injured after drinking, and (3) missing class or work due to drinking. For example, the odds of being hurt or injured as a result of drinking in the past 12 months were over 9 times higher among past-year binge drinkers compared with non-binge drinkers when gender, race/ethnicity, and ADO were statistically controlled. It is also worth noting that past-year binge drinking was associated with an increase in the odds of seriously thinking about suicide.

The second set of analyses was identical to the first, except that we also statistically controlled for past 2-week binge drinking by entering it as a covariate in each model. As can be seen in the bottom panel of Table 4, although 
Table 4. Multiple Logistic Regression Analyses of Past-Year Binge Drinking As a Predictor of Past Year Negative Consequences of Alcohol Use and Potential Alcohol Abuse: With and Without Controlling for Past 2-Week Binge Drinking

\begin{tabular}{|c|c|c|c|c|c|c|c|c|c|c|c|c|}
\hline & $\begin{array}{l}\text { Performed } \\
\text { poorly on } \\
\text { test }\end{array}$ & $\begin{array}{l}\text { Missed } \\
\text { class/ } \\
\text { work }\end{array}$ & $\begin{array}{l}\text { Driven while } \\
\text { under } \\
\text { influence }\end{array}$ & $\begin{array}{l}\text { Been } \\
\text { hurt or } \\
\text { injured }\end{array}$ & Vomited & $\begin{array}{l}\text { Been taken } \\
\text { advantage of } \\
\text { sexually }\end{array}$ & $\begin{array}{l}\text { Took advantage } \\
\text { of another } \\
\text { sexually }\end{array}$ & $\begin{array}{l}\text { Had V } \\
\text { unplanned } \\
\text { sex }\end{array}$ & $\begin{array}{l}\text { Were afraid you } \\
\text { might be } \\
\text { alcoholic }\end{array}$ & $\begin{array}{c}\text { Had } \\
\text { blackouts } \\
\text { (amnesia) }\end{array}$ & $\begin{array}{c}\text { Seriously } \\
\text { thought about } \\
\text { suicide }\end{array}$ & CAGE \\
\hline $\begin{array}{l}\text { Past year } \\
\text { binge } \\
\text { drinking }^{a} \\
\text { Controlling }\end{array}$ & $4.53^{* *}$ & $6.61^{* *}$ & $3.76^{* *}$ & $9.49^{* *}$ & $7.84^{* *}$ & $4.52^{* *}$ & $4.73^{* *}$ & $4.49^{* *}$ & $2.93^{* *}$ & $11.79^{* *}$ & $3.69^{* *}$ & $4.25^{* *}$ \\
\hline $\begin{array}{l}\text { Past year } \\
\text { binge } \\
\text { drinking }^{b}\end{array}$ & $2.52^{* *}$ & $3.04^{* *}$ & $2.47^{* * *}$ & $4.65^{* *}$ & $4.91^{* *}$ & $2.67^{* *}$ & $2.32 *$ & $2.24^{* *}$ & $1.69^{*}$ & $6.00^{* *}$ & $2.74^{*}$ & $2.63^{* *}$ \\
\hline
\end{tabular}

Note. Analyses based on $n=3,823$ past-year drinkers. Data weighted by gender and race/ethnicity.

${ }^{a}$ Adjusted odds ratios from multiple logistic regression analyses with past-year binge drinking as a predictor of past-year drinking consequences controlling for gender, race/ethnicity, and age of drinking onset.

${ }^{b}$ Adjusted odds ratios from multiple logistic regression analyses with past-year binge drinking as a predictor of past-year drinking consequences controlling for past 2-week binge drinking, gender, race/ethnicity, and age of drinking onset.

${ }^{*} p<0.05 .{ }^{* *} p<0.01$.

the associations were smaller in magnitude, past-year binge drinking continued to have statistically significant associations with all of the negative drinking outcomes, even after past 2-week binge drinking, gender, race/ ethnicity, and ADO were statistically controlled. These results are consistent with the hypothesis that the new past-year measure of binge drinking is independently associated with negative drinking outcomes.

\section{Past-Year Binge Drinking as a Correlate of Potential Alcohol Abuse}

One particularly important negative drinking outcome is potential alcohol abuse. The CAGE was used to identify college students who may be at risk for alcohol abuse. The results indicated that $23.5 \%$ of past-year drinkers $(23.5 \%$ of males and $23.6 \%$ of females) met CAGE screening criteria for alcohol abuse. As can be seen in Table 4, the results from multiple logistic regression analysis showed that past-year binge drinking was significantly associated with potential alcohol abuse as defined by the CAGE $(\mathrm{OR}=4.25, p<0.01)$ when gender, race/ethnicity, and ADO were statistically controlled. Even when past 2-week binge drinking was statistically controlled, the new past-year binge measure retained its significant association with potential alcohol abuse $(\mathrm{OR}=2.63, p<0.01)$.

\section{DISCUSSION}

The purpose of this study was to compare 2 measures of binge drinking: the standard measure, which typically defines binge drinking as the consumption of 5 (for a male)/4 (for a female) or more drinks in a row on at least 1 occasion during the past 2 weeks, and a new measure based on a definition of binge drinking as the consumption of 5 or more drinks (male) or 4 or more drinks (female) on at least 1 occasion during a 2 -hour period in the past year (NIAAA, 2004). The standard 2-week and new past-year measures were compared for overall and subgroup prevalence estimates and their associations with gender, race/ethnicity, and ADO. The hypothesis that the new past-year measure would predict negative drinking consequences even after controlling for past 2-week binge drinking was also tested.

\section{Comparison of Standard and New Measures of Binge Drinking}

As predicted, the 2 measures were highly correlated; had a considerable degree of concordance (about 78\%); and had similar patterns of association with gender, race/ ethnicity, and ADO. Yet, the 2 measures yielded different prevalence estimates, both overall and for subgroups defined by gender, race/ethnicity, and ADO. For example, the overall prevalence of past 2-week binge drinking was $53.1 \%$, whereas the overall prevalence rate of past-year binge drinking was $63.6 \%$. Clearly, the higher prevalence estimate yielded by the new past-year measure was due to the use of a longer past-year reference period. Indeed, we found that about $22 \%$ of those classified as past-year binge drinkers using the new past-year measure did not binge drink during the previous 2 weeks. This finding is consistent with the results reported by Vik et al. (2000), who used a 3-month reference period and found elevated rates of binge drinking compared with the standard 2-week reference period. Vik et al. suggested that the standard 2-week reference period may underestimate the prevalence of binge drinking, and the present findings support this conclusion.

Specification of a 2-hour duration for the drinking episode in the new past-year measure also influenced the classification of participants as binge drinkers or non-binge drinkers, such that about $10 \%$ of those who were categorized as binge drinkers using the standard 2-week measure were categorized as nonbinge drinkers using the new 
past-year measure. This finding indicates that the new past-year measure excludes those who consume $4 / 5$ drinks over extended periods of time (i.e., greater than 2 hours) from the binge drinker category. If in fact this pattern of consumption is not sufficient to raise BACs to the $0.08 \mathrm{~g} \%$ or above criterion, the findings suggest that the new past-year measure is potentially better able to discriminate between hazardous and less hazardous patterns of heavy drinking.

Previous research has documented strong associations between past 2-week binge drinking and alcohol-related problems (Perkins, 2002; Wechsler and Isaac, 1992; Wechsler et al., 1994, 2000, 2002). Although there was considerable concordance between the 2 measures in the percentage of participants identified as binge drinkers, our results showed that the new past-year measure remained a statistically significant predictor of most negative drinking consequences even when the standard 2-week measure was statistically controlled. The present findings are consistent with those from previous studies using the standard 2-week measure and further support the use of the new past-year measure as an indicator of problem drinking among college students. Of particular importance is the finding that those identified as binge drinkers by the new past-year measure were at elevated risk for possible alcohol abuse, controlling for past 2-week binge drinking. This result is consistent with previous work showing that binge drinking correlates with alcohol-dependence symptoms in college students (Knight et al., 2002; O'Neill et al., 2001) and noncollege samples (Midanik, 1999). Taken together, these results suggest that the new past-year measure captures a dimension of risky alcohol involvement in college students that is not fully assessed by the standard measure.

Comparisons with other studies that have used the past year time frame without specifying a 2-hour duration for the drinking episode are difficult because few studies have used the past year assessment period. The only large-scale study we are aware of that used the past year time frame is the 2001-2002 National Epidemiologic Survey on Alcohol and Related Conditions (NESARC; Dawson et al., 2004). Dawson et al. used the 2001-2002 NESARC and reported past year rates of binge drinking among college students that ranged from a low of $25.9 \%$ (among college students ages 18-20 who were living with parents) to a high of $54.1 \%$ (among college students ages $21-24$ who were living on campus). These rates compare with the rate of $63.6 \%$ in the present study. However, comparison between our rates and the NESARC rates may be misleading because the current study included only full-time undergraduate students at a 4-year school; in contrast, the NESARC included part-time students, graduate students, and students at 2-year schools, and there is evidence for lower rates of binge drinking among students at 2-year schools (Presley et al., 2002). Studies that include measures of binge drinking that differ only in the 2-hour duration of the drinking episode would help to address this limitation.
Race/Ethnicity, Gender, and Age of Onset Differences in the Prevalence of Binge Drinking

The results showed few differences between the standard 2-week and new past-year measures in the pattern of prevalence rates by gender, race/ethnicity, and ADO. Consistent with previous work (Dawson et al., 2004; Presley et al., 1996; Wechsler et al., 2002), relatively lower rates of past 2-week and past-year binge drinking were observed among Asians and Blacks compared with Whites and Hispanics. Genetic and cultural explanations have been proposed to account for ethnic group differences in rates of binge drinking. For example, previous research indicates that low rates of binge drinking among Asians are partially attributable to the greater prevalence of a genetic variation of the aldehyde dehydrogenase (ALDH2) gene (i.e., the ALDH2*2 allele) in Asian compared with Caucasian populations (Luczak et al., 2001). Further, the finding that Black students also had lower levels of binge drinking compared with white and Hispanic students is consistent with the hypothesis that blacks have more conservative norms and attitudes toward the use of alcohol (Galvan and Caetano, 2003). Recent evidence also indicates that, compared with White students, Blacks who attend college are less likely to engage in heavy drinking (Paschall et al., 2005).

The current results also showed elevated levels of past 2-week and past-year binge drinking among males, which replicates a consistent finding in the literature (Dawson et al., 2004; Wechsler et al., 1995, 2002; for a review, see Nolen-Hoeksema, 2004). However, this gender difference varied by race/ethnicity, with markedly higher rates of binge drinking for males compared with females among blacks compared with the other racial groups. These results suggest that lower rates of heavy drinking typically observed among black students in college-based research may reflect disproportionately lower drinking rates among black women. Findings also indicate that, with the exception of Blacks, gender differences in binge drinking are small among some racial groups. This observation is consistent with recent work showing that, along a variety of alcohol-related measures, gender differences have been decreasing in recent years (see Nolen-Hoeksema, 2004).

Prevalence rates of past 2-week and past-year binge drinking also varied as a function of ADO, with higher rates observed among students who began drinking before college. Participants who reported drinking onset in middle school and early high school were at a particularly high risk of binge drinking in college. The results from the present study are consistent with those from previous research that have linked an early ADO with a higher frequency of heavy episodic drinking (Muthen and Muthen, 2000) and with work showing that younger age at first intoxication predicts greater frequency of past 2-week binge drinking (Hingson et al., 2003). Early age of onset is a consistent predictor of subsequent alcohol involvement (Andersen et al., 2003; DeWit et al., 2000; 
Grant, 1998; Grant and Dawson, 1997; Grant et al., 2001; Muthen and Muthen, 2000; York et al., 2004), but the mechanisms underlying this association are unclear. Although our results do not address the underlying mechanisms, they do indicate that early age of onset is a robust correlate of binge drinking in college that does not vary by gender or race/ethnicity.

\section{Limitations}

There are several limitations to the present study. Although we drew a probability sample of college students, the study included students from only 1 university, and we do not know whether these findings generalize to other institutions. In addition, our comparisons of the 2 binge measures were limited by the fact that the new past-year binge scale differed from the standard 2-week measure in 2 respects: the specification of a 2-hour duration for the drinking episode, and the use of a longer (i.e., past year) time frame. Although our results suggested that both aspects of the new past-year binge measure influenced the resulting prevalence rates, the effects of the 2-hour window and the longer 12-month time frame were inherently confounded. As a result, our study was unable to distinguish the effects of the 2-hour window and the longer past-year time frame. While the higher rate of binge drinking yielded by the new past-year new measure relative to the past 2-week standard measure probably reflects the longer 1-year time frame, this rate may have been tempered by the 2-hour duration of the drinking episode.

Another limitation is that the new measure of binge drinking, while based on recent NIAAA recommendations, did not assess frequency of past-year binge drinking. This makes it impossible to distinguish frequent binge drinkers from those who binged only once in the past year. In addition, use of the longer past year time frame may have increased retrospection biases with respect to binge drinking episodes. Finally, nonresponse may have introduced some bias in the present study because $33 \%$ of the invited students did not elect to participate. To examine the possibility of nonresponse bias, we conducted a telephone follow-up survey of 159 randomly selected nonresponders. There were no differences in the prevalence rates of alcohol use, cigarette smoking, and past-year binge drinking between respondents and nonrespondents, which indicates that nonresponse bias was minimal.

\section{Conclusions and Implications}

Despite these limitations, our study has several important strengths. The use of a probability sample increases confidence in the generalization of the results to the larger population of the host university. Also, to our knowledge, this study is the first to provide comparisons between the 2-week standard binge measure and a version of the past year new binge measure based on recent NIAAA recommendations. Substantively, to our knowledge, this study is the first to document the prevalence of past-year binge drinking in college students using the new NIAAA-recommended measure. Our results indicated that over $60 \%$ of college students engaged in at least 1 episode of binge drinking during the previous year. This prevalence rate is particularly alarming in light of the negative consequences associated with binge drinking (Hingson, 2004; Wechsler et al., 2002) and the evidence that adolescent binge drinking predicts binge drinking well into adulthood (Jefferis et al., 2005).

An important task for future work is to incorporate multiple measures of alcohol misuse as the field attempts to develop a more universal measure of binge drinking. The present findings indicate that future studies would benefit by adding items that assess the duration of drinking episodes (Hingson, 2004) and their frequency (Presley and Pimentel, 2006). As noted earlier, studies that include binge measures that differ only in the 2-hour duration of the drinking episode would help to address the effects of drinking duration on rates of binge drinking. Also, more work is needed to document the associations between binge drinking and damage to other people besides the individual drinker (Perkins, 2002). Longitudinal research on the temporal ordering of the variables predictive of binge drinking and their associations with alcohol-related problems will be most informative for prevention and intervention efforts. In a recent analysis of data from the 2001 National Household Survey on Drug Abuse (NHSDA), Slutske (2005) found that 18\% of college students met criteria for a past year alcohol use disorder. The extent to which binge drinking increases the risk of alcohol use disorders among college students is an important question for longitudinal research.

\section{REFERENCES}

Agresti A (1996) An Introduction to Categorical Data Analysis. Wiley, New York, NY.

Andersen A, Due P, Holstein B, Iversen L (2003) Tracking drinking behaviour from age 15-19 years. Addiction 98:1505-1511.

Beirness DJ, Foss RD, Vogel-Sprott M (2004) Drinking on campus: self-reports and breath tests. J Stud Alcohol 65:600-604.

Beirness DJ, Foss RD, Vogel-Sprott MD (2005) Drinking on campus: self-reports and breath tests: response. J Stud Alcohol 66:439-440.

Boyd CJ, McCabe SE, d'Arcy H (2003) A modified version of the CAGE as an indicator of alcohol abuse and its consequences among undergraduate drinkers. Subst Abus 24:221-232.

Cahalan D, Cisin IH, Crossley HM (1969) American Drinking Practices: A National Study of Drinking Behavior and Attitudes. Rutgers Center of Alcohol Studies, New Brunswick, NJ.

Chassin L, Pitts SC, Prost J (2002) Binge drinking trajectories from adolescence to emerging adulthood in a high-risk sample: predictors and substance abuse outcomes. J Consult Clin Psychol 70:67-78.

Cisin IH, Cahalan D (1968) Comparison of abstainers and heavy drinkers in a national survey. Psychol Res Rep 24:10-22.

Connors GJ, Volk RJ (2003) Self-report screening for alcohol problems among adults, in Assessing Alcohol Problems: A Guide for Clinicians and Researchers, NIH Publication No. 03-3745, 2nd ed (Allen JP, Wilson VB eds), pp. 21-35. National Institute on Alcohol Abuse and Alcoholism, Bethesda, MD. 
Dawson DA (2003) Methodological issues in measuring alcohol use. Alcohol Res Health 27:18-29.

Dawson DA, Grant BF, Stinson FS, Chou PS (2004) Another look at heavy episodic drinking and alcohol use disorders among college and noncollege youth. J Stud Alcohol 65:477-488.

Dawson DA, Room R (2000) Towards agreement on ways to measure and report drinking patterns and alcohol-related problems in adult general population surveys: the Skarpo Conference overview. J Subst Abuse 12:1-20.

DeJong W (2001) Finding common ground for effective campus-based prevention. Psychol Addict Behav 15:292-296.

DeWit DJ, Adlaf EM, Offord DR, Ogborne AC (2000) Age at first alcohol use: a risk factor for the development of alcohol disorders. Am J Psychiatry 157:745-750.

Dimeff LA, Kilmer J, Baer JS, Marlatt GA (1995) Binge drinking in college. JAMA 273:1903-1904.

Ewing JA (1984) Detecting alcoholism: the CAGE Questionnaire. JAMA 252:1905-1907.

Galvan FH, Caetano R (2003) Alcohol use and related problems among ethnic minorities in the United States. Alcohol Res Health 27:87-94.

Grant BF (1998) The impact of a family history of alcoholism on the relationship between age at onset of alcohol use and DSM-IV alcohol dependence: results from the national longitudinal alcohol epidemiologic survey. Alcohol Health Res World 22:144-147.

Grant BF, Dawson DA (1997) Age at onset of alcohol use and its association with DSM-IV alcohol abuse and dependence: results from the National Longitudinal Alcohol Epidemiologic Survey. J Subst Abuse 9:103-110.

Grant BF, Stinson FS, Harford TC (2001) Age at onset of alcohol use and DSM-IV alcohol abuse and dependence: a 12-year follow-up. J Subst Abuse 13:493-504.

Ham LS, Hope DA (2003) College students and problematic drinking: a review of the literature. Clin Psychol Rev 23:719-759.

Harford TC, Wechsler H, Muthen BO (2002) The impact of current residence and high school drinking on alcohol problems among college students. J Stud Alcohol 63:271-279.

Hingson R (2004) Advances in measurement and intervention for excessive drinking. Am J Prev Med 27:261-263.

Hingson R, Heeren T, Jamanka A, Howland J (2000) Age of drinking onset and unintentional injury involvement after drinking. JAMA 284:1527-1533.

Hingson R, Heeren T, Winter M, Wechsler H (2005) Magnitude of alcohol-related mortality and morbidity among U.S. college students ages 18-24: changes from 1998 to 2001. Ann Rev Public Health 26: 259-279.

Hingson R, Heeren T, Zakocs R, Winter M, Wechsler H (2003) Age of first intoxication, heavy drinking, driving after drinking and risk of unintentional injury among U.S. college students. J Stud Alcohol 64:23-31.

Jaccard J (2001) Interaction Effects in Logistic Regression. Sage, Thousand Oaks, CA.

Jefferis BJMH, Power C, Manor O (2005) Adolescent drinking level and adult binge drinking in a national birth cohort. Addiction 100: 543-549.

Johnston LD, O'Malley PM, Bachman JG, Schulenberg JE (2005) Monitoring the Future National Survey Results on Drug Use, 1975-2004: Volume II, College Students and Adults Ages 19-45, NIH Publication No. 05-5728. National Institute on Drug Abuse, Bethesda, MD.

Knight JR, Wechsler H, Kuo M, Seibring M, Weitzman ER, Schuckit MA (2002) Alcohol abuse and dependence among U.S. college students. J Stud Alcohol 63:263-270.

Lange JE, Voas RB (2001) Defining binge drinking quantities through resulting blood alcohol concentrations. Psychol Addict Behav 15: 310-316.

Luczak SE, Wall TL, Shea SH, Byun SM, Carr LG (2001) Binge drinking in Chinese, Korean, and White college students: genetic and ethnic group differences. Psychol Addict Behav 15:306-309.
Maisto SA, Saitz R (2003) Alcohol use disorders: screening and diagnosis. Am J Addict 12:S12-S25.

Mayfield D, McLeod G, Hall P (1974) The CAGE questionnaire: validation of a new alcoholism screening instrument. Am J Psychiatry 131:1121-1123.

Midanik LT (1999) Drunkenness, feeling the effects and 5+ measures. Addiction 94:887-897.

Muthen BO, Muthen LK (2000) The development of heavy drinking and alcohol-related problems from ages 18 to 37 in a U.S. national sample. J Stud Alcohol 61:290-300.

Naimi TS, Brewer RD (2005) Correspondence: "binge" drinking and blood alcohol concentration. J Stud Alcohol 66:438.

National Institute on Alcohol Abuse and Alcoholism Task Force of the National Advisory Council on Alcohol Abuse and Alcoholism: A Call to Action: Changing the Culture of Drinking at U.S Colleges. National Institute on Alcohol Abuse and Alcoholism, National Institutes of Health, Bethesda, MD

National Institute on Alcohol Abuse and Alcoholism (2004) NIAAA Council Approves Definition of Binge Drinking, NIAAA Newsletter, No. 3. National Institute on Alcohol Abuse and Alcoholism, Bethesda, MD.

Nelson TF, Xuan Z (2005) Drinking on campus: self-reports and breath tests: comment. J Stud Alcohol 66:438-439.

Nolen-Hoeksema S (2004) Gender differences in risk factors and consequences for alcohol use and problems. Clin Psychol Rev 24:981-1010.

Office of Applied Studies (2003) The NSDUH Report: Alcohol Use and Risks among Young Adults by College Enrollment Status. Substance Abuse and Mental Health Services Administration, Rockville, MD.

O'Malley PM, Bachman JG, Johnston LD (1984) Period, age, and cohort effects on substance use among American youth, 1976-1982. Am J Public Health 74:682-688.

O'Neill SE, Parra GR, Sher KJ (2001) Clinical relevance of heavy drinking during the college years: cross-sectional and prospective perspectives. Psychol Addict Behav 15:350-359.

Paschall MJ, Bersamin M, Flewelling RL (2005) Racial/ethnic differences in the association between college attendance and heavy alcohol use: a national study. J Stud Alcohol 66:266-274.

Perkins HW (2002) Surveying the damage: a review of research on consequences of alcohol misuse in college populations. J Stud Alcohol 14 (suppl): 91-100.

Perkins HW, DeJong W, Linkenbach J (2001) Estimated blood alcohol levels reached by "binge" and "nonbinge" drinkers: a survey of young adults in Montana. Psychol Addict Behav 15:317-320.

Presley CA, Meilman PW, Cashin JR (1996) Alcohol and Drugs on American College Campuses: Use, Consequences, and Perceptions of the Campus Environment. Vol. IV: 1992-1994. The Core Institute, Southern Illinois University at Carbondale, Carbondale, IL.

Presley CA, Meilman PW, Leichliter JS (2002) College factors that influence drinking. J Stud Alcohol Suppl14:82-90.

Presley CA, Pimentel ER (2006) The introduction of the heavy and frequent drinker: a proposed classification to increase accuracy of alcohol assessments in postsecondary educational settings. J Stud Alcohol 67:324-331.

SAS Institute Inc (2004) SAS/STATS 9.1 User's Guide. SAS Institute Inc., Cary, NC.

Slutske WS (2005) Alcohol use disorders among U.S. college students and their non-college-attending peers. Arch Gen Psychiatry 62: $321-327$

Sobell LC, Sobell MB (2003) Alcohol consumption measures, in Assessing Alcohol Problems: A Guide for Clinicians and Researchers, 2nd ed (Allen JP, Wilson VB eds), pp. 75-99. U.S. Department of Health and Human Services, Bethesda, MD.

Stevens J (1996) Applied Multivariate Statistics for the Social Sciences, 3rd ed. Erlbaum, Mahwah, NJ.

Vik PW, Carrello PD, Tate SR, Field C (2000) Progression of consequences among heavy-drinking college students. Psychol Addict Behav 14:91-101. 
Wechsler H, Austin SB (1998) Binge drinking: the 5/4 measure. J Stud Alcohol 59:122-123.

Wechsler H, Davenport A, Dowdall G, Moeykens B, Castillo S (1994) Health and behavioral consequences of binge drinking: a national survey of students at 140 campuses. JAMA 272:1672-1677.

Wechsler H, Dowdall GW, Davenport A, Castillo S (1995) Correlates of college student binge drinking. Am J Public Health 85:921-926.

Wechsler H, Isaac N (1992) "Binge" drinkers at Massachusetts colleges: prevalence, drinking style, time trends, and associated problems. JAMA 267:2929-2931.

Wechsler H, Lee JE, Kuo M, Lee H (2000) College binge drinking in the 1990s: a continuing problem - results of the Harvard School of Public Health 1999 College Alcohol Study. J Am Coll Health 48:199-210.
Wechsler H, Lee JE, Kuo M, Seibring M, Nelson TF, Lee H (2002) Trends in college binge drinking during a period of increased prevention efforts: findings from 4 Harvard School of Public Health College Alcohol Surveys: 1993-2001. J Am Coll Health 50:203-217.

Wechsler H, Nelson TF (2001) Binge drinking and the American college student: what's five drinks? Psychol Addict Behav 15:287-291.

Weingardt KR, Baer JS, Kivlahan DR, Roberts LJ, Miller ET, Marlatt GA (1998) Episodic Heavy Drinking Among College Students: Methodological Issues and Longitudinal Perspectives. Psychol Addict Behav 12:155-167.

York JL, Welte J, Hirsch J, Hoffman JH, Barnes G (2004) Association of age at first drink with current alcohol drinking variables in a national general population sample. Alcohol Clin Exp Res 28:1379-1387. 